\title{
Angiostatic actions of capsicodendrin through selective inhibition of VEGFR2-mediated AKT signaling and disregulated autophagy
}

\author{
Christopher C. Pan ${ }^{1}$, Nirav Shah ${ }^{1}$, Sanjay Kumar ${ }^{1}$, Sarah E. Wheeler ${ }^{1}$ Jason \\ Cinti $^{1}$, Dale G. Hoyt ${ }^{1}$, Christine E. Beattie ${ }^{6}$, Min An ${ }^{6}$, Karthikeyan Mythreye ${ }^{2}$, L. \\ Harinantenaina Rakotondraibe ${ }^{3}$ and Nam Y. Lee ${ }^{1,4,5}$ \\ ${ }^{1}$ Division of Pharmacology, College of Pharmacy, The Ohio State University, OH, USA \\ 2 Department of Chemistry and Biochemistry, University of South Carolina, Columbia, SC, USA \\ ${ }^{3}$ Division of Medicinal Chemistry and Pharmacognosy, College of Pharmacy, The Ohio State University, OH, USA \\ ${ }^{4}$ Davis Heart Lung Research Institute, The Ohio State University, OH, USA \\ ${ }^{5}$ Comprehensive Cancer Center, The Ohio State University, $\mathrm{OH}$, USA \\ ${ }^{6}$ Department of Neuroscience, The Ohio State University, OH, USA \\ Correspondence to: Nam Y. Lee, email: lee.5064@osu.edu
}

Keywords: autophagy, AKT, VEGF, angiogenesis, endothelium

Received: April 15, $2016 \quad$ Accepted: April 29, $2016 \quad$ Published: May 11, 2016

\section{ABSTRACT}

Angiogenesis is the formation of new blood vessels from existing vasculature critical for embryonic development and vascular remodeling. Its dysregulation underlies numerous pathologic states ranging from ischemia to tumor growth and as such identifying new targeted- therapies is of significant interest for angiogenesisbased medicine. Here we evaluated the potential angiostatic properties of capsicodendrin (CPCD), a natural compound isolated from Cinnamosma macrocarpa, a plant belonging to the Malagasy Cinnamosma. CPCD potently inhibits endothelial proliferation, migration and capillary tube formation at nanomolar to low micromolar concentrations without inducing cytotoxic effects. We show that CPCD directly inactivates VEGFR2 and downstream AKT signaling, thereby strongly inducing autophagy as determined by increased expression of beclin 1, autophagy-related gene (Atg) 3, Atg5 and LC3 cleavage. Ectopic AKT overexpression counteracts the inhibitory effects of CPCD on proliferation and capillary tubule formation. Importantly, CPCD treatment in vivo inhibits sprouting angiogenesis as evidenced by strongly reduced intersegmental vessel (ISV) sprouting and subintestinal vessel (SIV) formation during zebrafish embryonic development, and correlates with increased presence of LC3II along the ISVs despite overall reduced vasculature. These findings demonstrate CPCD as a potent inhibitor of the VEGFR2/AKT pathway at nanomolar concentrations and inducer of autophagy-related angiostatic effects.

\section{INTRODUCTION}

Angiogenesis is the formation of new blood vessels from pre-existing vasculature, a highly coordinated multi-step process involving endothelial matrix degradation, migration, proliferation and capillary tube formation [1]. Numerous therapeutic strategies are aimed at revascularizing ischemic or damaged tissues, and conversely, inhibiting angiogenesis in tumors and proliferative retinopathies [2-4]. While many anti-angiogenic treatments demonstrate limited longterm efficacy due to evasive or intrinsic resistance, improvements on progression-free survival among patients with various degrees of metastatic and advanced cancers are still achieved when combined with conventional chemotherapy, and anti-angiogenic therapies may have clear benefits in normalizing tumor vessels for better drug delivery resistance [5-7]. 
A number of new classes of therapeutic agents are being developed, and natural products represent an important source of novel drug leads and the basis for many rational synthetic designs [8-12]. A growing number of small molecules have been identified as promising agents with dozens in preclinical and clinical trials including the flavonoids. These and other natural products and metabolites act through multiple interdependent mechanisms including apoptosis, matrix metalloproteinase-2 (MMP2) downregulation, and quite often, by suppressing vascular endothelial growth factor (VEGF) gene expression [13]. Interestingly, while their multitude of inhibitory targets could certainly work in favor of reducing the development of resistance by cancer cells, concerns of unpredictable or undesirable off-target effects remain a significant challenge in clinical settings.

As the plant species in the Canellaceae family endemic to Madagascar, three species- Cinnamosma fragrans, C. macrocarpa and C. madagascariensis are all widely used to alleviate a range of symptoms including malaria and fatigue, and is further believed in Malagasy traditional medicine to possess antiviral and anticancer properties $[14,15]$. Preliminary studies of isolated $C$. macrocarpa derivatives identified capsicodendrin (CPCD) as a lead compound that was highly soluble and stable in aqueous environment, and capable of exerting cytostatic activity against a broad spectrum of cancer cell types including murine Leukemia cells (L1210/0), human T-lymphocyte cells (Molt4/C8 and CEM/0), HeLa and HT29 cells at sub-micromolar ranges [16].

Many chemotherapeutic agents possess antiangiogenic properties as they produce cytotoxic or cytostatic effects by targeting cellular pathways that promote apoptosis or autophagy [17, 18]. The latter process has clear roles in various cellular and pathologic states, although its major function in angiogenesis is somewhat contentious [19-23]. Indeed, many studies have shown that autophagy inhibits angiogenic vasculature, whereas others have suggested its key role in neovessel formation. Several natural compounds capable of inducing autophagy-mediated inhibition of angiogenesis and cell death have already been reported, and in many instances, appear to target a broad range of cellular pathways including VEGF gene expression [17, 18, 22].

In the present study, we tested CPCD as a lead compound for potential anti-angiogenic activity and defined its mechanism of action. We report that CPCD has distinct autophagy-related angiostatic effects in vitro and in vivo.

\section{RESULTS}

Prior to investigating the potential angiostatic effects of CPCD, its chemical structure, molecular mass and stability were determined by NMR and mass spectrometry, and the overall purity of the isolated molecule from $C$. macrocarpa verified by total ion chromatography (see Supplementary material online, Figure S1). We first tested the effects of CPCD using the MTT growth assay in mouse embryonic endothelial cell line (MEEC) treated with or without increasing concentration of CPCD for up to $72 \mathrm{~h}$. Here, a moderate to significant growth-inhibition was observed at concentrations ranging from $100 \mathrm{nM}$ to 2 $\mu \mathrm{M}$ at $48 \mathrm{~h}$ (Figure 1A), a result that was recapitulated in a parallel study involving human microvascular endothelial cell 1 (HMEC1) (Figure 1B). Similar results were obtained using crystal violet colorimetric assay as a read-out of cell proliferation, and found a largely concentration-dependent growth-inhibition was observed over the course of $72 \mathrm{~h}$ upon drug treatment (Figure 1C). To test whether apoptosis contributed to the overall growth inhibition, annexin- $\mathrm{V}$ staining was performed in MEECs and isolated primary mouse aortic endothelial cells (MAECs). Compared to control chloroquine treatment, CPCD did not promote apoptosis even at higher concentrations up to $72 \mathrm{~h}$ (Figure $1 \mathrm{D}$ and graph, see Supplementary material online, Figure S2), suggesting that CPCD exerts cytostatic effects on endothelial cells. Next, we assessed the effects of CPCD on cell motility using the Boyden transwell chamber system. Relative to control, CPCD inhibited endothelial migration in a concentration-dependent manner (Figure 1E and graph), a finding that was consistent with the dosedependent inhibition of capillary tube formation in threedimensional matrigel assay using MEECs and MAECs (Figure 1F and graph, see Supplementary material online, Figure S3A). Taken together, these results indicated that CPCD acts as a potent angiostatic compound in vitro.

To define the underlying mechanism, we screened for angiogenic pathways affected by CPCD including ERK, p38, PLC $\gamma$, among others (Figure 2). Despite its potent cytostatic effects, CPCD had minimal impact on mitogenic signaling such as ERK and p38 (Figure 2A). Instead, there was a notable concentration-dependent AKT inactivation (Figure 2A and graph). Despite its key role in cell survival, AKT signaling attenuation had little impact on caspase- 3 cleavage even at up to $5 \mu \mathrm{M}$ (Figure 2B), and hence agreed with our annexin- $\mathrm{V}$ results.

Aside from cell survival, AKT mediates a number of other cellular processes including migration, metabolism and autophagy. Given that AKT is a suppressor of autophagy in most systems, we examined whether the CPCD-dependent AKT inactivation promotes autophagy by measuring the relative levels of autophagic markers including beclin1, Atg3, Atg5 and microtubule-associated protein light chain 3 (LC3) (Figure 2C). A 2 h treatment with increasing concentrations of CPCD in both MEECs and HMEC1 resulted in elevated levels of beclin1, Atg3 and Atg5 expression (Figure 2C and 2D). Moreover, there was a corresponding decrease in inhibitory beclin 1 phosphorylation by AKT, strongly suggesting that CPCD is an inducer of autophagy (Figure 2C). Surprisingly, the final step of autophagy involving LC3-I conversion 

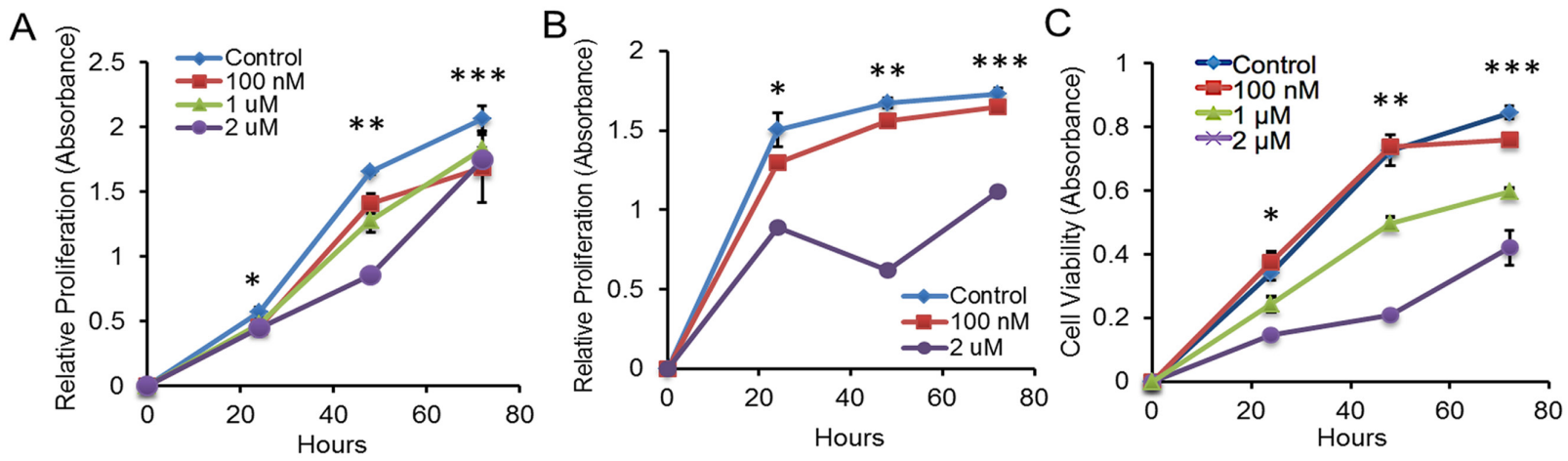

D
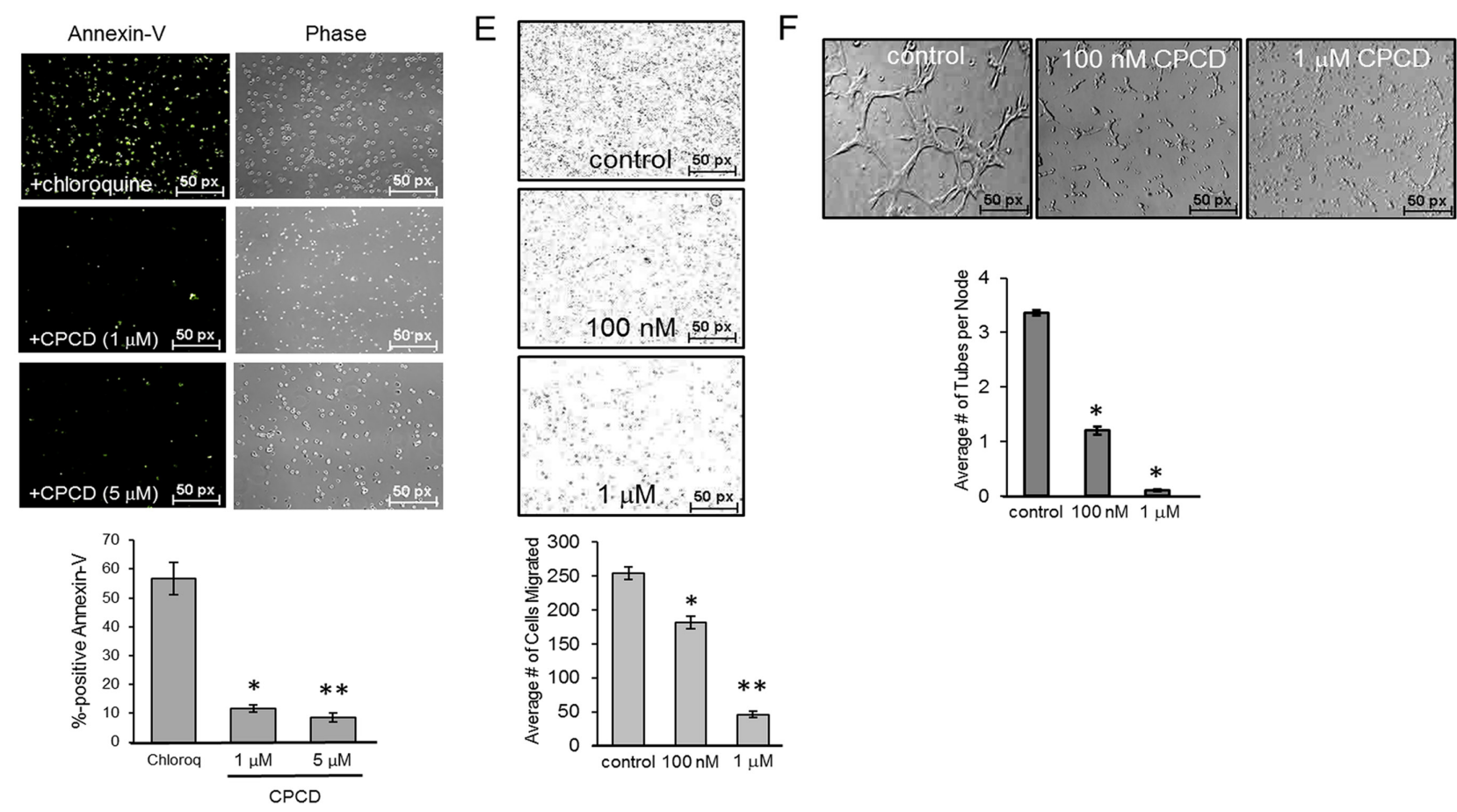

Figure 1: CPCD attenuates endothelial proliferation, migration, and capillary tube formation. A. MTT growth assay of MEECs treated with CPCD $(100 \mathrm{nM}, 1 \mu \mathrm{M}, 2 \mu \mathrm{M})$ for $24 \mathrm{~h} ., 48 \mathrm{~h}$., and $72 \mathrm{~h}$. Presented data are an average readings from 6 independent experiments. ANOVA analysis of MTT assay readings at $24 \mathrm{~h} ., 48 \mathrm{~h}$., and $72 \mathrm{~h}$. are as follows: $P=0.000135, P=2.33 \times 10^{-14}, P=0.003$. Student's $T$-Test analyses are as follows: $* P<0.001$ for control $v s 100 \mathrm{nM}$, control $v s 1 \mu \mathrm{M}$, control $v s 2 \mu \mathrm{M}, * * P<0.00005$ for control vs $100 \mathrm{nM}$, control vs $1 \mu \mathrm{M}$, control vs $2 \mu \mathrm{M}$, ***P<0.01 for control vs $100 \mathrm{nM}$, control $v s 1 \mu \mathrm{M}$, and control $v s 2 \mu \mathrm{M}$. B. MTT growth assay of HMECs treated with CPCD ( $100 \mathrm{nM}, 1 \mu \mathrm{M}, 2 \mu \mathrm{M})$ for $24 \mathrm{~h}$., $48 \mathrm{~h}$., and $72 \mathrm{~h}$. Presented data are an average readings from 5 independent experiments. ANOVA analysis of MTT assay readings at $24 \mathrm{~h} ., 48 \mathrm{~h} .72 \mathrm{~h}$. are as follows: $P=4.38 \times 10^{-20}, P=2.039 \times 10^{-18}, P=3.09 \times$ $10^{-12}$. Student's $T$-Test analyses are as follows: $* P<0.05$ for control $v s 100 \mathrm{nM}, * * P<0.0003$ for control $v s 100 \mathrm{nM}$, control $v s 1 \mu \mathrm{M}$, and control $v s 2 \mu \mathrm{M}$. C. Crystal violet growth assay of MEECs treated with CPCD (100 nM, $1 \mu \mathrm{M}, 2 \mu \mathrm{M})$ for $24 \mathrm{~h} ., 48 \mathrm{~h}$., and $72 \mathrm{~h}$. Presented data are average readings from 4 independent experiments. ANOVA analysis of crystal violet readings at $24 \mathrm{~h} ., 48 \mathrm{~h}$. , and $72 \mathrm{~h}$. are as follows: $P=2.048 \times 10^{-5}, P=1.85 \times 10^{-8}, P=5.51 \times 10^{-11}$. Student's $T$-Test analyses are as follows: ${ }^{*} P<0.05$ for control $v s 100 \mathrm{nM}$, control vs $1 \mu \mathrm{M}$, and control vs $2 \mu \mathrm{M}, * * P<9.1 \times 10^{-7}$ for control vs $1 \mu \mathrm{M}$, and control vs $2 \mu \mathrm{M}, * * * P<0.004$ for control $v s 100 \mathrm{nM}$, control vs 1 $\mu \mathrm{M}$, and control vs $2 \mu \mathrm{M}$. D.. Representative images of MEEC Annexin V staining upon chloroquine $(1 \mu \mathrm{M})$ and $\mathrm{CPCD}$ treatment $(1 \mu \mathrm{M}$, $5 \mu \mathrm{M}$ ) for $48 \mathrm{~h}$. followed by quantification of the percentage of Annexin $V$ positive cells per field. 10 random fields for each condition were analyzed from 4 independent experiments. ANOVA analysis are as follows: $P=4.08 \times 10^{-9}$. Student's $T$-Test analyses are as follows: $* P$ $=4.09 \times 10^{-6}, * * P=2.55 \times 10^{-6}$. E. Representative images of MEEC transwells upon CPCD treatment $(100 \mathrm{nM}, 1 \mu \mathrm{M})$ for $16 \mathrm{~h}$. followed by quantification of the average number of cells migrated. For each condition, 12 random fields were digitally imaged and counted from 4 independent experiments. ANOVA analysis are as follows: $P=4.3 \times 10^{-15}$. Student's $T$-Test analyses are as follows: $* P=3.6 \times 10^{-5}, * * P$ $=1.2 \times 10^{-12}$. F. Representative images of three-dimensional Matrigel-induced capillary tubules for MEECs treated with CPCD $(100 \mathrm{nM}$, $1 \mu \mathrm{M}$ ) for $6 \mathrm{~h}$. followed by quantification of the average number of capillary tubes per node. For each condition, eight random fields were digitally imaged and analyzed from 4 independent experiments. ANOVA analyses are as follows: $P=2.02 \times 10^{-11}$. Student's $T$-Test analyses are as follows: $* P=3.1 \times 10^{-7}, * * P=1.2 \times 10^{-9}$. 
to LC3-II significantly decreased in a dose-dependent manner in our biochemical analysis and time-course study as monitored by immunofluorescence staining of LC3positive autophagosomes (Figure 2C; fourth panel and Figure $3 \mathrm{~A}$ and graph). As LC3-II itself is degraded during autophagy in the lysosome, we speculated that CPCD markedly accelerates LC3-II degradation. To test this, we pre-treated MEECs with a lysosomal inhibitor chloroquine in the presence or absence of CPCD and found a striking increase in LC3-II autophagasome vesicles (Figure 3B; arrows). Similar results were obtained in our biochemical analysis wherein the basal level of LC3-II rose only modestly upon lysosomal inhibition while CPCD treatment drastically raised LC3-II levels under the same condition in MEECs and primary MAECs (Figure 3C and $3 \mathrm{D}$, and see Supplementary material online, Figure S3). Collectively, these results indicated that CPCD induces endothelial autophagy by targeting AKT activation.

We next sought to determine the mechanism by which CPCD blocks AKT activation and downstream signaling. Given its potent angiostatic effects, we tested whether this compound targets vascular endothelial growth factor receptor 2 (VEGFR2), a major upstream activator of AKT in the endothelial system. Indeed, a time-course experiment upon drug treatment followed by a biochemical analysis of receptor autophosphorylation revealed VEGFR2 inactivation within minutes, particularly at Tyr1175, which mediates the recruitment and activation of the PI3-kinase and subsequent AKT signaling (Figure 4A) [24]. To determine whether CPCD directly targets VEGFR2, the intracellular segment comprising the receptor kinase and C-terminal autophosphorylation domain was purified in baculovirus/insect cell system and subjected to an in vitro kinase assay in the presence or absence of CPCD pretreatment. Upon initiating the kinase reaction with ATP, there was rapid kinase activation as evidenced by tyrosine autophosphorylation at $5 \mathrm{~min}$, and continued to rise at $15 \mathrm{~min}$ (Figure 4B, see Supplementary
A

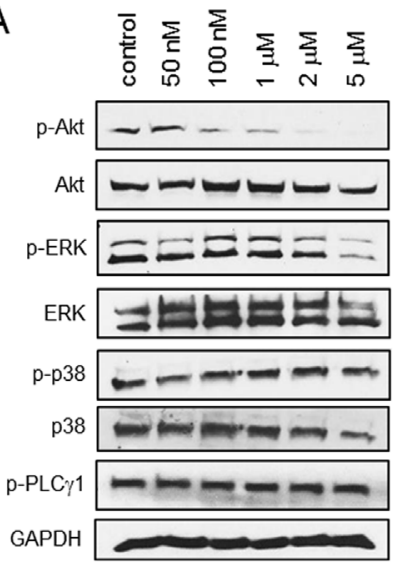

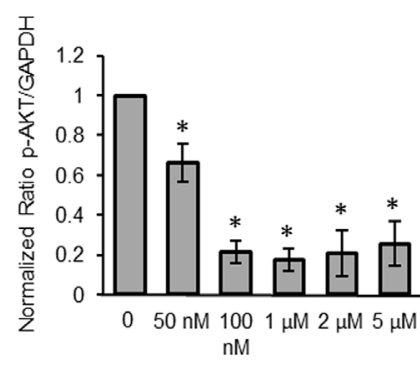

C

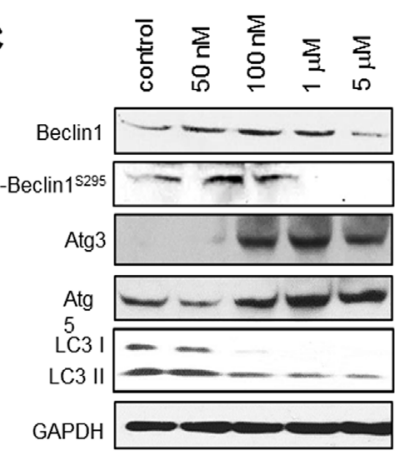

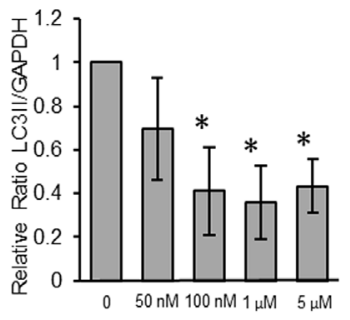

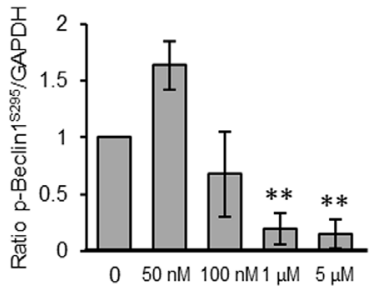

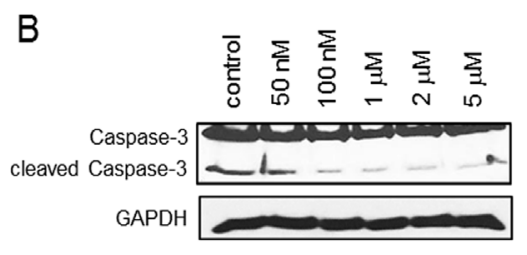
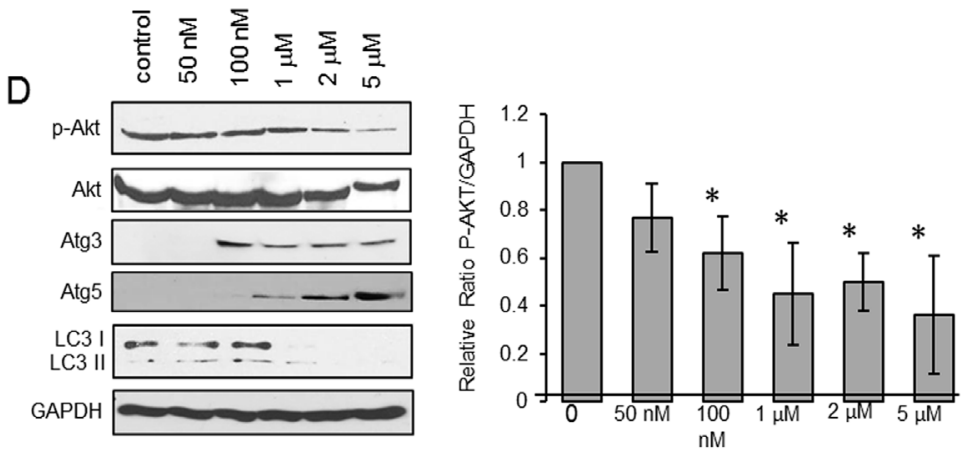

Figure 2: CPCD induces autophagy by impairing Akt activation. A. Western analysis of p-Akt protein levels in MEECs treated with CPCD ( $50 \mathrm{nM}, 100 \mathrm{nM}, 1 \mu \mathrm{M}, 2 \mu \mathrm{M}, 5 \mu \mathrm{M}$ ) for $2 \mathrm{~h}$. followed by densitometry analysis of p-Akt levels normalized to GAPDH from three independent experiments. Student's $T$-Test analyses are as follows: ${ }^{*} P<0.04$. B. Western analysis of caspase- 3 cleavage in MEECs treated with CPCD $(50 \mathrm{nM}, 100 \mathrm{nM}, 1 \mu \mathrm{M}, 2 \mu \mathrm{M}, 5 \mu \mathrm{M})$ for $2 \mathrm{~h}$. C. Western analysis of autophagic markers in MEECs treated with CPCD $(50 \mathrm{nM}, 100 \mathrm{nM}, 1 \mu \mathrm{M}, 2 \mu \mathrm{M}, 5 \mu \mathrm{M})$ for $2 \mathrm{~h}$. followed by densitometry analysis of LC3 II levels and p-Beclin1 $1^{\mathrm{s} 295}$ levels normalized to GAPDH from three independent experiments. Student's $T$-Test analyses are as follows: ${ }^{*} P<0.04,{ }^{*} * P<0.005$. D. Western analysis of autophagic markers in MEECs and HMECs treated with CPCD $(50 \mathrm{nM}, 100 \mathrm{nM}, 1 \mu \mathrm{M}, 2 \mu \mathrm{M}, 5 \mu \mathrm{M})$ for $2 \mathrm{~h}$. followed by densitometry analysis of p-Akt levels normalized to GAPDH from three independent experiments. Student's $T$-Test analyses are as follows: ${ }^{*} P<0.05$. 
material online, Figure S4A). In contrast, pre-incubating the purified protein with CPCD prior to ATP addition prevented receptor autophosphorylation, indicating that the small molecule acts as an inhibitor of VEGFR2 kinase (Figure 4B). Since VEGFR2 kinase inactivation helps explain how CPCD attenuates AKT signaling, we next evaluated the role of AKT-targeting in endothelial proliferation (Figure 4C). Here, CPCD treatment resulted in reduced proliferation in control MEECs, whereas AKT overexpression enhanced basal proliferation and counteracted the growth-inhibitory effects of CPCD (Figure 4C). Moreover, the matrigel capillary tube assay yielded a similar pattern in which, relative to control, AKT overexpressing MEECs resisted the overall angiostatic effects of CPCD (Figure 4D and graph). Taken together, these results strongly supported AKT as a major inhibitory target of CPCD during angiogenesis.

Lastly, we evaluated the angiostatic effects in vivo by monitoring the effects of CPCD on $\operatorname{Tg}($ flil:eGFP) zebrafish during embryonic development (Figure 5) [27]. Here, fish embryos were grown in petri dish containing $2 \mu \mathrm{M}$ of CPCD. Compared to control, CPCD treatment exerted potent anti-angiogenic effects within the first 24 hours post-fertilization (hpf), as indicated by reduced vascular sprouting of intersegmental vessels (ISV) (Figure 5A arrows). Upon 48 hpf, significant angiogenic defects were evident including abnormal subintestinal vessel (SIV) formation and markedly diminished SIV sprouts (Figure 5B; a-d arrows). Importantly, while CPCD treatment significantly diminished the overall sprouting of the ISVs relative to control, more LC3-II vesicles were present along the defective ISVs (Figure 5C arrows), supporting our in vitro data that $\mathrm{CPCD}$ inhibits angiogenesis by disrupting the regulation of endothelial autophagy.
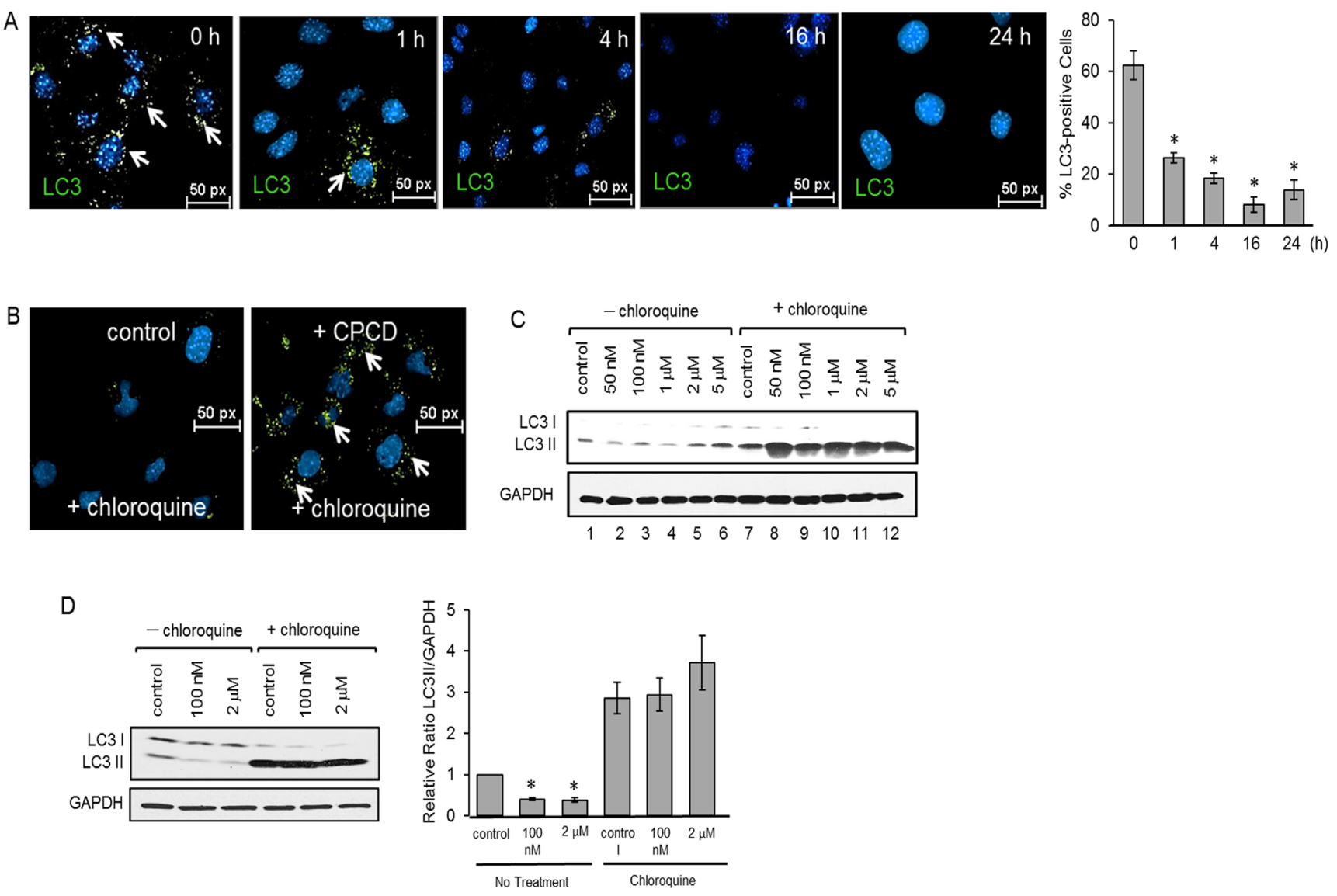

Figure 3: CPCD rapidly enhances LC3 I/II degradation. A. Representative images of MEECs stained for LC3 I/II after treatment with CPCD (100 nM) for $1 \mathrm{~h} ., 4 \mathrm{~h} ., 16 \mathrm{~h}$., and $24 \mathrm{~h}$. followed by quantification of percentage of LC3 I/II positive cells. For each condition, 13 random fields were digitally imaged and analyzed. ANOVA analyses are as follows: $P=2.77 \times 10^{-9}$. Student's $T$-Test analyses are as follows: $* P<0.000144$. B. Representative images of MEECs treated with CPCD (100nM) and chloroquine $(1 \mu \mathrm{M})$ for $2 \mathrm{~h}$. and stained for LC3 I/II. C. Western analysis of LC3 cleavage in MEECs cotreated with CPCD (50 nM, $100 \mathrm{nM}, 1 \mu \mathrm{M}, 2 \mu \mathrm{M}, 5 \mu \mathrm{M})$ and chloroquine $(1 \mu \mathrm{M})$ for $2 \mathrm{~h}$. D) Western analysis of LC3 cleavage in MAECs cotreated with CPCD (100 nM, $2 \mu \mathrm{M})$ and chloroquine $(1 \mu \mathrm{M})$ for $2 \mathrm{~h}$. followed by densitometry analysis of LC3 II levels normalized to GAPDH from three independent experiments. ANOVA analyses are as follows: $P<0.0001$. Student's $T$-Test analyses are as follows: $* P<0.0003$. 


\section{DISCUSSION}

Our work defines novel cytostatic properties of a natural compound isolated from C. macrocarpa. Aside from its use as traditional medicine for numerous ailments, CPCD demonstrates selective inhibition of the VEGFR2 kinase and downstream AKT activation. Our data further supports enhanced autophagy as the major mechanism by which it inhibits angiogenesis, although other ATK-related cellular functions such as metabolism still remain to be explored.

Considerable progress has been made over the years with targeted anti-cancer and anti-angiogenic therapies, and natural compounds have undoubtedly played an integral role in drug-discovery efforts. Indeed, there is an ever-growing inventory of small molecule inhibitors derived from natural products, many of which are currently under preclinical or clinical evaluations [9]. However, achieving high potency and selectivity on the desired target continue to be the principal challenge even among many promising drugs in the pipeline. Along these lines, our study demonstrates CPCD as a promising lead compound given its in vitro efficacy at nanomolar concentrations. Still, despite its potent angiostatic effects, CPCD showed signs of potential off-target cardiovascular effects in vivo, as we observed a dose-dependent decrease in the heart rate of developing zebrafish embryos at 72 hpf (see Supplementary material online, Figure S4B). Although no gross changes were evident in terms of the overall size of the embryos ( $\sim 1-2 \mathrm{~mm}$ diameter) or the heart structure compared to control (data not shown), additional studies are required to fully characterize the cardiovascular effects of CPCD in developing versus adult vertebrate systems.

While many natural compounds previously have shown inhibitory properties of VEGF signaling, the precise mechanisms are often unclear as they broadly target multiple cellular pathways including VEGF, VEGR2, AKT, MAPKs, hypoxia-inducible factors (HIFs) and many others [11]. Hence, it is noteworthy that
A

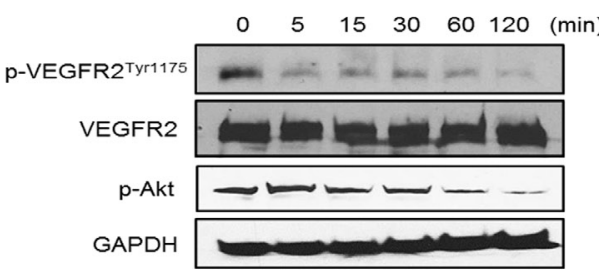

$\mathrm{B}$

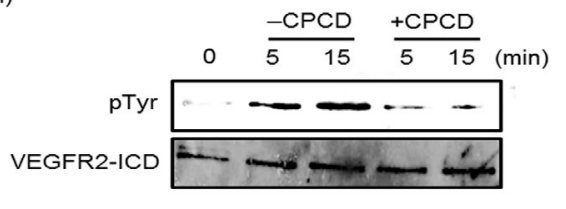

C

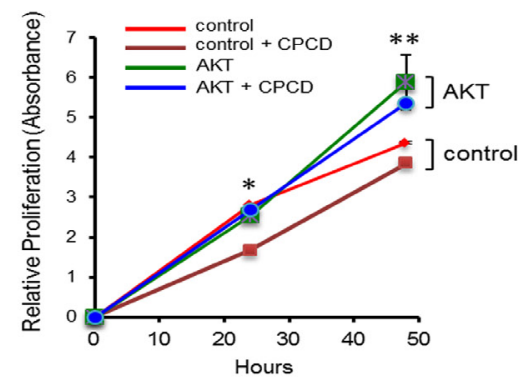

D
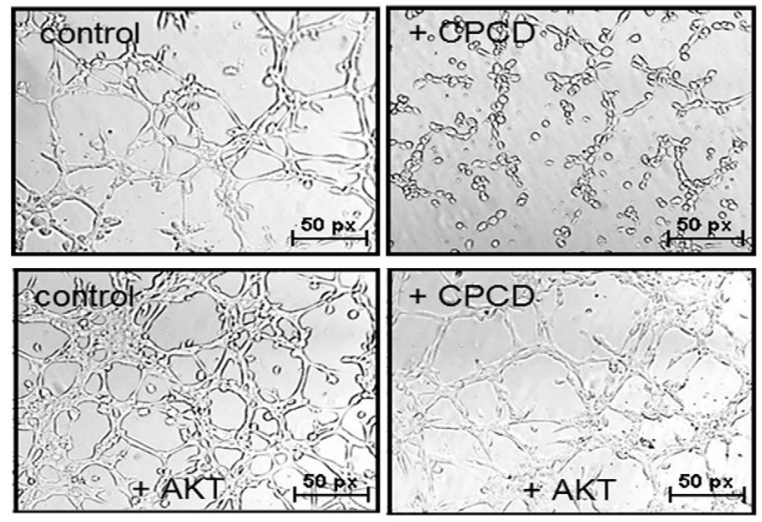

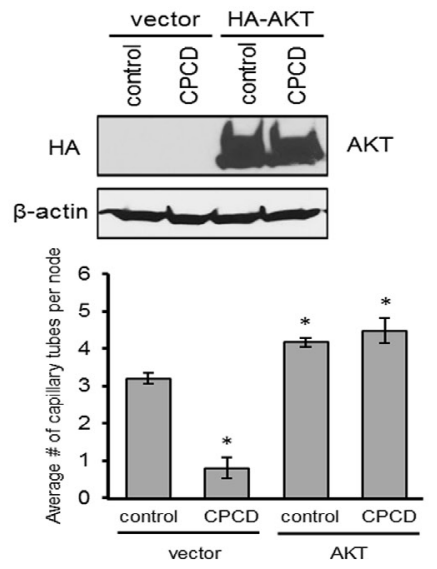

Figure 4: CPCD impairs Akt activation by inhibiting VEGFR2 ${ }^{\text {Tyr1175 }}$ phosphorylation. A. Western analysis of p-VEGFR2 $2^{\text {Tyr1175 }}$ levels and p-Akt levels in MEECs treated with CPCD (100 nM) for the indicated time (5 min., 15 min., 30 min., 60 min., 120 min.) B. Western analysis of VEGFR2 intracellular tyrosine phosphorylation upon $5 \mu \mathrm{M} \mathrm{CPCD}$ treatment ( $5 \mathrm{~min} ., 15 \mathrm{~min}$.) C. Crystal violet growth assay of MEECs overexpressing Akt and treated with CPCD $(2 \mu \mathrm{M})$ for $24 \mathrm{~h}$. and $48 \mathrm{~h}$. Presented data are average readings from 4 independent experiments. ANOVA analyses are as follows: $* P=7.4 \times 10^{-12}, * * P=0.005$. Student's $T$-Test analyses are as follows: $* P=2.0$ x $10^{-9}$ for control vs control $+\mathrm{CPCD},{ }^{* * P}=0.01$ for control $v s$ control + CPCD. D. Representative images of three-dimensional Matrigelinduced capillary tubules for MEECs overexpressing Akt after CPCD treatment $(100 \mathrm{nM})$ for $6 \mathrm{~h}$. followed by quantification of the average number of capillary tubes per node and western analysis of HA-Akt overexpression. 10 random fields were digitally imaged and analyzed for each condition from 4 independent experiments. ANOVA analyses are as follows: ${ }^{*} P=5.38 \times 10^{-10}$. Student's $T$-Test analyses are as follows: * $P=2.49 \times 10^{-6}, * * P<0.0005$. 
CPCD appears to be more selective in terms of directly targeting the VEGFR2 kinase and attenuating AKT activity at nanomolar concentrations while ERK and other mitogenic signaling remain unperturbed (Figure 2 and 4A, 4B). Although it is not clear whether CPCD also inhibits other tyrosine kinases, we found that it did not inhibit the activation of at least one other receptor tyrosine kinase, the insulin receptor, in endothelial cells under similar conditions (data not shown). Understanding the molecular mechanism by which CPCD inhibits VEGFR2 autophosphorylation would require direct structureactivity relationship studies involving in silico docking and biophysical characterization.

Autophagy is a dynamic process of subcellular degradation that mediates cell survival under nutrientdeprivation, hypoxia and many other stress-related conditions. The precise role of autophagy in angiogenesis is contentious with many conflicting reports indicating either anti-angiogenic or pro-angiogenic properties. In our system, CPCD proved to be a strong autophagy inducer capable of enhancing the major autophagy-related components including beclin1, Atg3 and 5, as well as producing high-rate turnover of LC3-II degradation. More importantly, our in vivo findings establish a link between the angiostatic effects of CPCD and increased autophagy within defective sprouting vessels. Despite the controversy, basal autophagy is presumably required for angiogenesis, as it clears damaged organelles, longlived proteins and has other protective functions during a time of high metabolic demand. In contrast, excessive self-digestion dramatically increases the formation of reactive oxygen species (ROS) that induces cytotoxicity in many cell types including the endothelial system. Our data suggests that CPCD tips this critical balance towards excessive autophagy that impairs critical endothelial functions during angiogenesis.
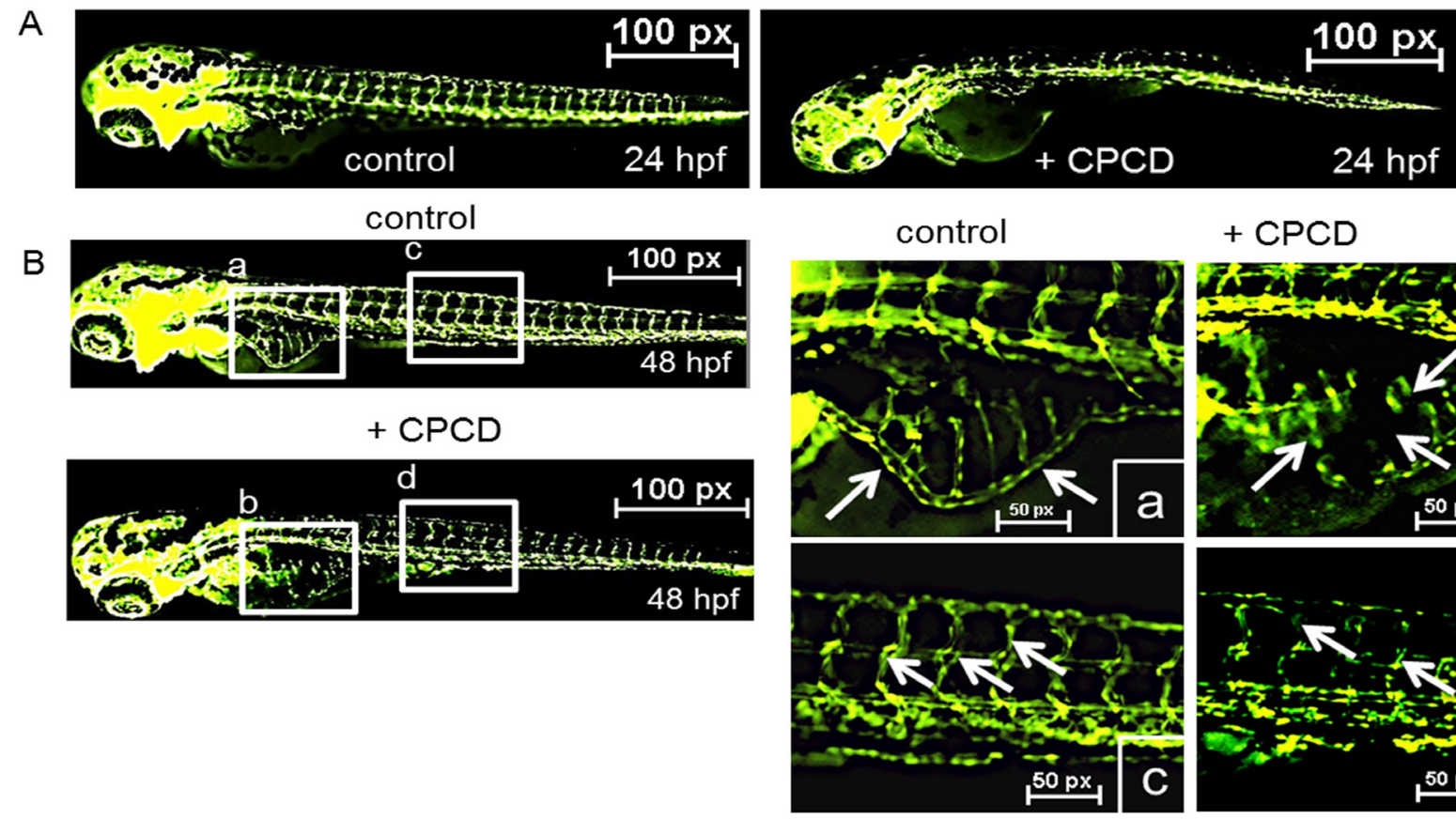

+ CPCD

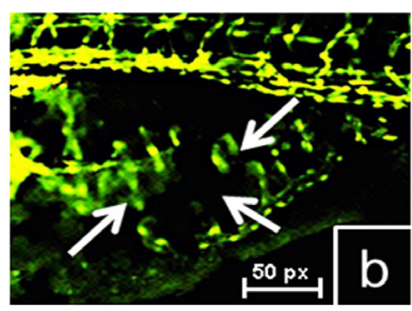

$48 \mathrm{hpf}$
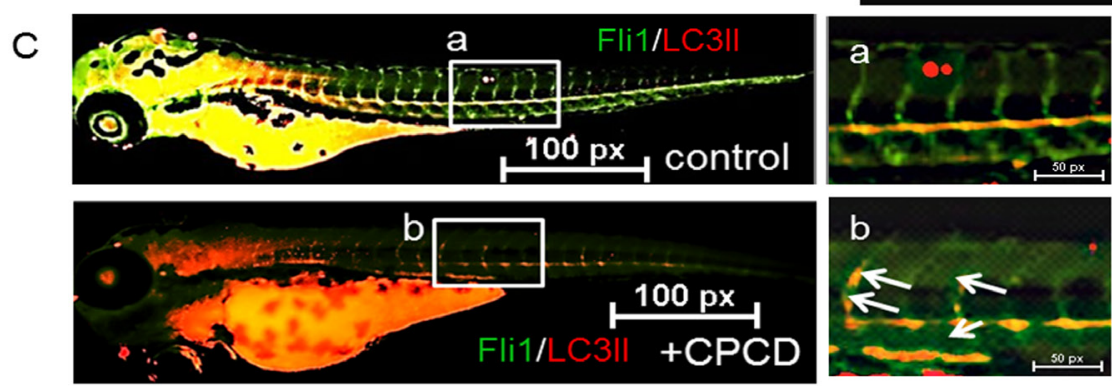

Figure 5: CPCD negatively regulates sprouting angiogenesis in vivo. A. Representative images ofTg(fli1:eGFP) embryos treated with CPCD $(2 \mu \mathrm{M})$ for $24 \mathrm{~h}$. B. CPCD $(2 \mu \mathrm{M})$ treated embryos displayed impaired sprouting of ISVs, an absence of the DLAV, and a reduction of the SIV. 30 embryos were analyzed to determine the angiogstatic effects of CPCD. C. Representative images of zebrafish embryos stained for LC3II (red). 
Taken together, our work defines the potent angiostatic role of $\mathrm{CPCD}$, a promising lead compound that serves as a basis for the development of new derivatives and combination therapy studies in preclinical settings.

\section{MATERIALS AND METHODS}

\section{Cell culture, plasmids, transfections, and antibodies}

All mouse and zebrafish studies were performed in accordance with the guidelines provided by the Institutional Animal Care and Use Committee (IACUC) at The Ohio State University and the NIH guidelines (Guide for the care and use of laboratory animals). All established endothelial cell lines used were of comparable passage number of 10-20. Mouse embryonic endothelial cell line (MEECs) was derived from mice at E9 and cultured as previously described $[23,25]$. Briefly, C57BL/6 mice purchased from Jackson Laboratories (Maine). Mice were euthanized according to the NIH and The Ohio State University approved guidelines, involving intraperitoneal injection of $300 \mathrm{mg} / \mathrm{kg}$ body weight sodium pentobarbital. MEECs were maintained in MCDB-131 medium (Invitrogen) supplemented with $2 \mathrm{mM} \mathrm{L}$-glutamine, 1 $\mathrm{mM}$ sodium pyruvate, $15 \%$ fetal bovine serum, $100 \mu \mathrm{g} /$ $\mathrm{ml}$ heparin, $25 \mu \mathrm{g} / \mathrm{ml}$ endothelial cell growth supplement. Human microvascular endothelial cell line (HMEC-1) was purchased through ATCC and maintained in MCDB131 medium supplemented with $10 \%$ fetal bovine serum, $1 \mu \mathrm{g} / \mathrm{ml}$ hydrocortisone (Sigma), $10 \mathrm{ng} / \mathrm{ml}$ epidermal growth factor (Sigma), and $2 \mathrm{mM}$ L-glutamine. Primary mouse aortic endothelial cells (MAECs) were isolated and cultured as we previously described and used at low passage numbers $(<10)$ [26]. Briefly, C57BL/6 mice purchased from Jackson Laboratories. Mice were euthanized according to the NIH and The Ohio State University approved guidelines, involving intraperitoneal injection of $300 \mathrm{mg} / \mathrm{kg}$ body weight sodium pentobarbital. MAECs were maintained in 40\% DMEM, 40\% HAM, $20 \%$ fetal bovine serum, $30 \mathrm{mg} / \mathrm{ml}$ endothelial cell growth supplement, $100 \mu \mathrm{g} / \mathrm{ml}$ heparin. Transfections were achieved by using Lipofectamine 2000 as described according to manufacturer protocol (Invitrogen). HAAkt construct was a generous gift from Dr. Frederick W. Quelle, University of Iowa. Basement matrigel matrix was obtained from BD Bioscience. The lysosome inhibitor chloroquine, $\beta$-actin, and anti-HA antibodies were purchased from Sigma-Aldrich. P-Beclin $1^{\mathrm{S} 295}$ was purchased from Abcam. Anti-phosphotyrosine antibody, PY20, was purchased from Millipore. The following antibodies were all purchased from Cell Signaling: phospho-Akt (no. 13038), total Akt (no. 4685), phosphoERK (no. 9101), total ERK (no. 9102), phospho-p38 (no.
4511), total p38 (no. 8690), phospho-PLC $\gamma 1$ (no. 14008), GAPDH (no. 5174), Beclin-1 (no. 3495), Atg3 (no. 3415), Atg5 (no. 12994), LC3 A/B (no. 4108), Cleaved Caspase-3 (no. 9664), p-VEGFR2 ${ }^{\text {Tyr1175 }}$ (no. 2478), total VEGFR2 (no. 9698), Annexin V-FITC early apoptosis detection kit (no. 6592).

\section{Immunofluorescence}

MEECs and MAECs were grown overnight on coverslips and treated with CPCD $(100 \mathrm{nM})$ alone or CPCD $(100 \mathrm{nM})$ in the presence of chloroquine $(1 \mu \mathrm{M})$ for the indicated duration. Following treatment, cells were fixed with $4 \%$ paraformaldehyde. Cells were permeabilized in $0.1 \%$ Triton X-100 in PBS for 3-10 min, then blocked with $5 \%$ bovine serum albumin in PBS containing $0.05 \%$ Triton X-100 for $20 \mathrm{~min}$. All primary Abs were incubated at room temperature for $1 \mathrm{~h}$ unless noted otherwise. LC3 A/B Ab was used to detect autophagosome clusters. Following primary antibody incubation, cells were incubated with appropriate flurophore conjugated secondary antibodies (AlexaFluor) at room temperature for $30 \mathrm{~min}$. Cells were co-stained with DAPI (Sigma) immediately before immunofluorescence microscopy analyses (Nikon Eclipse Ti). Autophagy levels were quantified by counting the number of autophagosome-positive cells.

\section{Annexin V-FITC staining}

MEECs and MAECs were grown overnight 10 $\mathrm{cm}$ plates and treated with CPCD $(1 \mu \mathrm{M}, 5 \mu \mathrm{M})$ for 48 $\mathrm{h}$. and $72 \mathrm{~h}$. Following treatment, cells were trypsinized, pelleted, and resuspsended with $500 \mu \mathrm{l}$ of $1 \mathrm{X}$ Annexin V Binding Buffer. Following Annexin V Binding Buffer, 1 $\mu \mathrm{l}$ of Annexin V-FITC conjugated antibody and $12.5 \mu \mathrm{l}$ of propidium iodide were added. Cells were incubated in the dark on ice for 10 mins and then analyzed by immunofluorescence microscopy and ImageJ.

\section{CPCD isolation and molecular characterization}

Powdered C. fragrans was extracted with methanol and then purified by column chromatography on silica gel (solvent: hexane-ethyl acetate gradient). Purification of fractions were characterized by ${ }^{1} \mathrm{H}$ and ${ }^{13} \mathrm{C}$ NMR on Varian Unity $600 \mathrm{NMR}$ using $\mathrm{CDCl}_{3}$ as a solvent. Mass spectra were recorded on JEOL JMS AX-500 spectrometer. Column chromatography was carried out on Sephadex LH-20 (Amersham) and silica gel. Preparative HPLC was performed using a reversed-phase column. 


\section{Endothelial tube formation}

MEECs or MEECs transiently overexpressing the wild type mouse Akt were plated on a 24-well plate coated with $200 \mu \mathrm{l}$ of matrigel basement matrix (Corning) at 140,000 cells/well in the presence of CPCD at the indicated doses. $1 \mathrm{~h}$ following plating, growth medium was removed and $200 \mu \mathrm{l}$ of matrigel was added. $30 \mathrm{~min}$ following the addition of the matrigel basement matrix, $300 \mu \mathrm{l}$ of growth medium containing the indicated doses of CPCD was added. Endothelial tubes were digitally imaged and quantified by counting the number of branches per node.

\section{Transwell migration assays}

MEECs were seeded in the upper chamber of a transwell filter in complete growth media containing CPCD at various doses, coated both at the top and bottom with gelatin. Cells were allowed to migrate for 12 $\mathrm{h}$ toward the lower chamber. Cells that migrated to the bottom surface of the filter were fixed, stained, and then digitally imaged and counted.

\section{Crystal violet and MTT growth assay}

MEEC control and cells transiently overexpressing Akt were plated at 15,000 in 12-well plates and treated with different doses of CPCD. Cells were fixed at different time points (4\% paraformaldehyde in PBS for $15 \mathrm{~min}$ ). Following fixation, cells were washed with $1 \times$ water and stained with $0.1 \%$ crystal violet for $20 \mathrm{~min}$. Cells were washed $3 \times$ with water and allowed to air dry for $30 \mathrm{~min}$. Cells were destained using crystal violet de-staining solution ( $10 \%$ acetic acid, $50 \%$ methanol, $\left.40 \% \mathrm{H}_{2} 0\right)$ for $20 \mathrm{~min}$, and the optical density was read at $590 \mathrm{~nm}$ in a microplate reader. Similarly, the MTT assay MEECs were plated at 15,000 in 12-well plates and treated with different doses of CPCD for 24, 48 and $72 \mathrm{~h}$. Following incubation, the media was aspirated and replaced with $500 \mu \mathrm{l}$ of MTT $(5 \mathrm{mg} / \mathrm{mL})$ for $3 \mathrm{~h}$ at $37 \mathrm{C}$. MTT was aspirated and dissolving buffer $(4 \mathrm{mM} \mathrm{HCl}, 0.1 \% \mathrm{NP} 40$ in isopropanol) was added and the absorbance was measured at $590 \mathrm{~nm}$ in a microplate reader

\section{Cloning and generation of recombinant baculovirus}

Gene encoding the intracellular domain (ICD) of VEGFR2 (790-1356 amino acids) was cloned into pFastBac1 vector (Life Technologies) between the NotI and XhoI restriction sites upon PCR amplification using a forward hexahistidine-encoding primer(5-GCGCGG CCGCGCCATGGATGCACCACCACCACCACCAC
AAGCGGGCCAATGAGGGG-3) and a reverse primer (5-CCGCTCGAGTTAAACAGG AGGAGAGCT-3). pFastBac1-VEGFR2-ICD plasmid was transformed into DH10Bac competent cells for production of bacmid DNA. Isolated bacmid DNA was transfected into Sf21 insect cells using Cellfectin II reagent (Invitrogen) according to protocols provided by manufacturer. Purified recombinant viruses were amplified with three rounds of infection in $\mathrm{Sf} 21$ cells grown at $27 \mathrm{C}$ using a multiplicity of infection (MOI) of 0.1. Viral supernatants were harvested 48-72 h post infection.

\section{Purification of recombinant protein}

Sf21 cells were infected with the baculovirus at MOI of 2. After $48 \mathrm{~h}$ post-infection, cells were harvested by centrifugation at $1000 \mathrm{~g}$ for $3 \mathrm{~min}$, gently washed with resuspension buffer (20 mM HEPES pH 7.4, $0.5 \mathrm{M} \mathrm{NaCl}$, $250 \mathrm{mM}$ sucrose, protease inhibitors [ $5 \mu \mathrm{g} / \mathrm{ml}$ aprotinin, 5 $\mu \mathrm{g} / \mathrm{ml}$ leupeptin, $2 \mu \mathrm{M}$ pepstatin A, $1 \mathrm{mM}$ PMSF]). Cells were resuspended with sucrose-free resuspension buffer and then lysed by sonication on ice. After sonication, cell lysate was centrifuged at $11,000 \mathrm{rpm}$ for $20 \mathrm{~min}$ prior to being supplemented with $0.05 \%$ Triton X-100 and then loaded onto a cobalt resin column (Thermo Scientific) preequilibrited with wash buffer (20 mM HEPES pH 7.4, 0.5 $\mathrm{M} \mathrm{NaCl}, 15 \mathrm{mM}$ imidazole, $0.05 \% \mathrm{TX}-100$ ). The column was washed with at least 20 column-volumes of wash buffer before elution with elution buffer (20mM HEPES $\mathrm{pH}$ 7.4, $0.5 \mathrm{M} \mathrm{NaCl}, 150 \mathrm{mM}$ imidazole, $0.05 \%$ TX$100)$ and $0.5 \mathrm{ml}$ fractions were collected and checked for protein concentration prior to being pooled and dialyzed extensively against 20mM HEPES (pH 7.4), $100 \mathrm{mM}$ $\mathrm{NaCl}$ at $4 \mathrm{C}$.

\section{In vitro protein tyrosine kinase assay}

Kinase assays were typically performed at $25 \mathrm{C}$ by pre-incubating $0.5 \mu \mathrm{M}$ of purified VEGFR2-ICD protein with $1 \mathrm{mM} \mathrm{MnCl}$ for $5 \mathrm{~min}$ in kinase assay buffer (20 $\mathrm{mM}$ HEPES at $\mathrm{pH} 7.4,150 \mathrm{mM} \mathrm{NaCl}$ ) and then $100 \mu \mathrm{M}$ ATP was added. The reactions were stopped after the noted period (5 and $15 \mathrm{~min}$ ) with SDS-PAGE sample buffer. Protein reactions were resolved by SDS-PAGE and immunoblotted with VEGFR2 (Cell signaling) and antiphosphotyrosine (PY20, Millipore).

\section{Zebrafish vascular sprouting during embryonic development}

$\operatorname{Tg}(f l i 1: e G F P)$ zebrafish embryos were obtained from Dr. Christine Beattie under the auspices of the NINDS P30 Zebrafish Genetics Core at the Ohio State University and grown in $10 \mathrm{~cm}$ petri dish filled fish water containing $2 \mu \mathrm{M}$ CPCD. 24 and 48 hours post 
fertilization, Fli1:EGFP zebrafish embryos were treated with tricaine $(160 \mu \mathrm{g} / \mathrm{mL})$ and digitally analyzed using immunofluorescence microscopy (Nikon Eclipse Ti). For immunostaining, $48 \mathrm{~h}$. embryos were fixed with $4 \%$ paraformaldehyde for $2 \mathrm{~h}$. and placed in $100 \%$ methanol for $2 \mathrm{~h}$ at $-20^{\circ} \mathrm{C}$. Embryos were then blocked using blocking buffer 1x PBST, 10\% heat-inactivated fetal bovine serum, $2 \%$ bovine serum albumin) for 1 h. at room temperature. Following blocking, embryos were incubated with LC3 A/B primary antibody (Cell Signaling) at $4{ }^{\circ} \mathrm{C}$ overnight. Following primary antibody incubation, embryos were incubated with anti-rabbit flurophore conjugated secondary antibody for $4 \mathrm{~h}$. at room temperature. Relative LC3 A/B levels and localization were analyzed using immunofluorescence microscopy (Nikon Eclipse Ti).

\section{Zebrafish embryo heart rate analysis}

Transgenic zebrafish were grown in $10 \mathrm{~cm}$ petri dish filled fish water containing $1 \mu \mathrm{M}$ and $5 \mu \mathrm{M}$ CPCD. At 24, 48 and $72 \mathrm{~h}$ hpf zebrafish hearts were visualized using immunofluorescence microscopy and heart rates were determined by counting the number of heartbeats per minute.

\section{Statistics}

Statistical analysis was performed using both student $t$-test and one-way anova. Data are presented as mean \pm SEM. Student $t$-test was performed to determine statistical significance for densitometry analysis of western blots. Statistical significance for all other experiments was determined using one-way ANOVA. $P$ values of $<0.05$ were considered as significant.

\section{ACKNOWLEDGMENTS}

We would like to thank Dr. Christine Beattie and Dr. Min An (Department of Neuroscience, The Ohio State University) for providing us with the transgenic zebrafish.

\section{CONFLICTS OF INTEREST}

The authors declare no conflict of interest.

\section{GRANT SUPPORT}

This work was supported by the National Cancer Institute at the National Institute of Health [CA178443 to N.Y.L.] and National Institute of General Medical Sciences [5P20GM109091 to M.K.] and National Institute of Neurological Disorders and Stroke [P30 NS045758 to C.E.B]

\section{Editorial note}

This paper has been accepted based in part on peerreview conducted by another journal and the authors' response and revisions as well as expedited peer-review in Oncotarget

\section{REFERENCES}

1. Carmeliet P. Angiogenesis in life, disease and medicine. Nature. 2005; 438:932-936.

2. Eichmann A and Simons M. VEGF signaling inside vascular endothelial cells and beyond. Curr Opin Cell Biol. 2012; 24:188-193.

3. Welti J, Loges S, Dimmeler S and Carmeliet P. Recent molecular discoveries in angiogenesis and antiangiogenic therapies in cancer. J Clin Invest. 2013; 123:3190-3200.

4. Weis SM and Cheresh DA. Tumor angiogenesis: molecular pathways and therapeutic targets. Nature Medicine. 2011; 17:1359-1370.

5. Ebos JM and Kerbel RS. Antiangiogenic therapy: impact on invasion, disease progression, and metastasis. Nat Rev Clin Oncol. 2011; 8:210-221.

6. Mackey JR, Kerbel RS, Gelmon KA, McLeod DM, Chia SK, Rayson D, Verma S, Collins LL, Paterson AH, Robidoux A and Pritchard KI. Controlling angiogenesis in breast cancer: a systematic review of anti-angiogenic trials. Cancer Treat Rev. 2012; 38:673-688.

7. Tol J, Koopman M, Cats A, Rodenburg CJ, Creemers GJ, Schrama JG, Erdkamp FL, Vos AH, van Groeningen CJ, Sinnige HA, Richel DJ, Voest EE, Dijkstra JR, VinkBorger ME, Antonini NF, Mol L, et al. Chemotherapy, bevacizumab, and cetuximab in metastatic colorectal cancer. N Engl J Med. 2009; 360:563-572.

8. Sulaiman RS, Basavarajappa HD and Corson TW. Natural product inhibitors of ocular angiogenesis. Exp Eye Res. 2014; 129:161-171.

9. Newman DJ and Cragg GM. Natural products as sources of new drugs over the 30 years from 1981 to 2010. J Nat Prod. 2012; 75:311-335.

10. Burnett J, Newman B and Sun D. Targeting cancer stem cells with natural products. Curr Drug Targets. 2012; 13:1054-1064.

11. Sagar SM, Yance D and Wong RK. Natural health products that inhibit angiogenesis: a potential source for investigational new agents to treat cancer-Part 1. Curr Oncol. 2006; 13:14-26.

12. Ejaz S, Anwar K, Taj R and Ashraf M. A novel link between angiogenesis and natural products: Anti-angiogenic effects of Opuntia dillenii. Cent Eur J Biol. 2014; 9:298-308.

13. Wang Z, Dabrosin C, Yin X, Fuster MM, Arreola A, Rathmell WK, Generali D, Nagaraju GP, El-Rayes B, Ribatti D, Chen YC, Honoki K, Fujii H, Georgakilas 
AG, Nowsheen S, Amedei A, et al. Broad targeting of angiogenesis for cancer prevention and therapy. Semin Cancer Biol. 2015.

14. Harinantenaina $\mathrm{L}$ and Takaoka S. Cinnafragrins A-C, dimeric and trimeric drimane sesquiterpenoids from Cinnamosma fragrans, and structure revision of capsicodendrin. J Nat Prod. 2006; 69:1193-1197.

15. Harinantenaina L, Matsunami K, Otsuka H, Kawahata M, Yamaguchi K and Asakawa Y. Secondary metabolites of Cinnamosma madagascariensis and their alpha-glucosidase inhibitory properties. J Nat Prod. 2008; 71:123-126.

16. Harinantenaina L, Asakawa $\mathrm{Y}$ and De Clercq E. Cinnamacrins A-C, cinnafragrin D, and cytostatic metabolites with alpha-glucosidase inhibitory activity from Cinnamosma macrocarpa. J Nat Prod. 2007; 70:277-282.

17. Sui X, Chen R, Wang Z, Huang Z, Kong N, Zhang M, Han W, Lou F, Yang J, Zhang Q, Wang X, He C and Pan H. Autophagy and chemotherapy resistance: a promising therapeutic target for cancer treatment. Cell Death Dis. 2013; 4:e838.

18. Choi KS. Autophagy and cancer. Exp Mol Med. 2012; 44:109-120.

19. Nussenzweig SC, Verma S and Finkel T. The role of autophagy in vascular biology. Circ Res. 2015; 116:480488.

20. Du J, Teng RJ, Guan T, Eis A, Kaul S, Konduri GG and Shi Y. Role of autophagy in angiogenesis in aortic endothelial cells. Am J Physiol Cell Physiol. 2012; 302:C383-391.

21. Yang SY, Kim NH, Cho YS, Lee H and Kwon HJ. Convallatoxin, a dual inducer of autophagy and apoptosis, inhibits angiogenesis in vitro and in vivo. PLoS One. 2014; 9:e91094.
22. Kumar S, Guru SK, Pathania AS, Kumar A, Bhushan S and Malik F. Autophagy triggered by magnolol derivative negatively regulates angiogenesis. Cell Death Dis. 2013; 4:e889.

23. Pan CC, Kumar S, Shah N, Bloodworth JC, Hawinkels LJ, Mythreye K, Hoyt DG and Lee NY. Endoglin Regulation of Smad2 Function Mediates Beclin1 Expression and Endothelial Autophagy. The Journal of biological chemistry. 2015; 290:14884-14892.

24. Holmqvist K, Cross MJ, Rolny C, Hagerkvist R, Rahimi N, Matsumoto T, Claesson-Welsh L and Welsh M. The adaptor protein shb binds to tyrosine 1175 in vascular endothelial growth factor (VEGF) receptor-2 and regulates VEGFdependent cellular migration. The Journal of biological chemistry. 2004; 279:22267-22275.

25. Pece-Barbara N, Vera S, Kathirkamathamby K, Liebner S, Di Guglielmo GM, Dejana E, Wrana JL and Letarte M. Endoglin null endothelial cells proliferate faster and are more responsive to transforming growth factor beta1 with higher affinity receptors and an activated Alk1 pathway. The Journal of biological chemistry. 2005; 280:2780027808.

26. Huang H, McIntosh J and Hoyt DG. An efficient, nonenzymatic method for isolation and culture of murine aortic endothelial cells and their response to inflammatory stimuli. In Vitro Cell Dev Biol Anim. 2003; 39:43-50.

27. Lawson, N. D., \& Weinstein, B. M. In Vivo Imaging of Embryonic Vascular Development Using Transgenic Zebrafish. Dev Biol. 2002; 248. 307-318. 